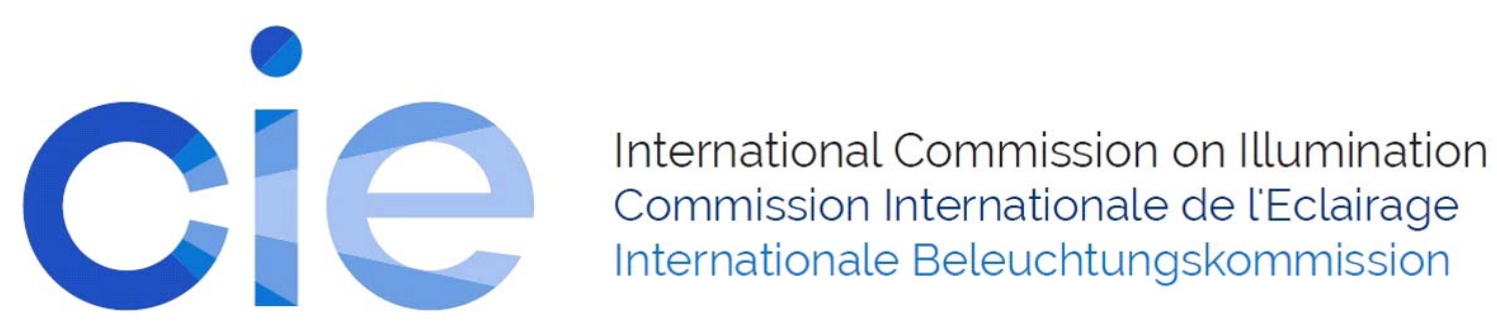

PO004

\title{
COGNITIVE PERFORMANCE EVALUATION UNDER CONTROLLED DAYLIGHT LEVELS AT DIFFERENT INDOOR TEMPERATURES
}

\author{
Giorgia Chinazzo et al.
}

DOI 10.25039/x46.2019.PO004

from

CIE x046:2019

Proceedings

of the

29th CIE SESSION

Washington D.C., USA, June 14 - 22, 2019

(DOI 10.25039/x46.2019)

The paper has been presented at the 29th CIE Session, Washington D.C., USA, June 14-22, 2019. It has not been peer-reviewed by CIE.

\section{(C) CIE 2019}

All rights reserved. Unless otherwise specified, no part of this publication may be reproduced or utilized in any form or by any means, electronic or mechanical, including photocopying and microfilm, without permission in writing from CIE Central Bureau at the address below. Any mention of organizations or products does not imply endorsement by the CIE.

This paper is made available open access for individual use. However, in all other cases all rights are reserved unless explicit permission is sought from and given by the CIE.

CIE Central Bureau

Babenbergerstrasse 9

A-1010 Vienna

Austria

Tel.: +4317143187

e-mail: ciecb@cie.co.at

www.cie.co.at 


\title{
COGNITIVE PERFORMANCE EVALUATION UNDER CONTROLLED DAYLIGHT LEVELS AT DIFFERENT INDOOR TEMPERATURES
}

\author{
Giorgia Chinazzo ${ }^{1}$, Jan Wienold ${ }^{1}$, Marilyne Andersen ${ }^{1}$ \\ ${ }^{1}$ Ecole polytechnique fédérale de Lausanne (EPFL), Lausanne, SWITZERLAND \\ giorgia.chinazzo@epfl.ch
}

DOI 10.25039/x46.2019.PO004

\begin{abstract}
This paper details the results of an experimental study investigating the influence of daylight illuminance levels and indoor temperatures on objective and subjective performance evaluations, investigated through paper-based tasks and questionnaires, respectively. Experiments were conducted in an office-like test room with controllable indoor temperature and easy-to-change glazing visible transmittance. A total of 84 participants took part in the experiment, performed only under clear sky conditions to avoid variations in illuminance. In a randomized order, participants were exposed to three daylight illuminance levels (on average 140,610 and 1440 lux) at one of three temperature conditions: 19,23 and $27^{\circ} \mathrm{C}$. Results show that objective performance outcomes were not affected by daylight illuminance or temperature, nor by their interactions, but only by the order of the test presentation (indicating a learning effect). Of the subjective responses, only perceived concentration was affected by daylight conditions, with a self-reported lower concentration under the low illuminance level.
\end{abstract}

Keywords: Daylight, Illuminance, Indoor temperature, Cognitive performance.

\section{Introduction}

Cognitive performance, defined as the extent to which a particular goal is achieved after an activity has been carried out, can be influenced by many factors such as motivation, type of task, personal control and personal characteristics, as well as by the indoor environmental factors (i.e., light, temperature, noise and indoor air quality) (Al Horr et al., 2016; ClementsCroome, 2006). Considering that performance is one of the inputs in the estimation of productivity, defined as the economic output of a person per unit time, then a change in performance of office workers would correlate directly to the financial outcome of an organization. For this reason, many studies have been conducted to investigate the impact of indoor environmental factors on human performance, focusing on one factor at a time (Baron et al., 1992; Furnham and Strbac, 2002; Heschong Mahone Group, 2003; Lan and Lian, 2009; Reinten et al., 2017; Valančius and Jurelionis, 2013; Wargocki, 1998). However, building occupants are exposed simultaneously to multiple factors and their performance might depend not only on the presence of individual factors but also on their combination (Torresin et al., 2018). Only few studies exist on the effects of the simultaneous presence of multiple indoor stimuli on objective or subjective performance (Balazova et al., 2007; Hygge and Knez, 2001; Knez and Hygge, 2002; Liebl et al., 2012; Lin, 2014; Löfberg et al., 1975; Veitch, 1990). To the best of the authors' knowledge, no study exists on the effect of interaction between temperature and daylight illuminance levels on cognitive performance. Similarly to what reported in the only study on light and temperature interactions with the use of electric light (Löfberg et al., 1975), high daylight illuminance conditions might lead to higher performance results, but only in neutral thermal condition. The study of the combination of daylight levels with indoor thermal conditions could further expand our understanding of the impact of indoor factors on human performance, potentially explaining some past contradicting or null results reported when indoor factors effects on cognitive performance were studied in isolation.

Towards this end, this paper presents the results of a controlled study investigating subjective performance evaluations and paper-based tasks performed under three daylight illuminance levels $(140 \pm 20,610 \pm 90$ and $1440 \pm 180$ lux, average and standard deviation values measured on the horizontal plane) at three different indoor temperatures (19, 23 and $27^{\circ} \mathrm{C}$ ). 
This study is part of a larger research investigation aiming at understanding interaction effects between daylight and indoor temperatures (Chinazzo, 2019). More specifically, one of these investigations focused on daylight illuminance levels, and was conducted to investigate the cross-modal effect of daylight illuminance on thermal responses (both perceptual and physiological), the cross-modal effect of indoor temperature on visual perception of daylight illuminance, as well as the combined effect of daylight illuminance and indoor temperature on overall comfort and performance (Chinazzo et al., 2019a, 2019b). This paper strictly focuses on the performance results, analyzing whether changes of performance could be observed in relation to daylight illuminance levels, temperatures and their interactions. Participants' performance was analyzed with two types of indicators, using objective performance measurements (paper-based tasks) and subjective performance evaluations through a questionnaire.

\section{Method}

Experimental investigations were carried out in an office-like test room $(3.05 \times 6.55 \mathrm{~m}$, presenting a north and a south opening) with controllable indoor temperature and easy-tochange glazing visible transmittance thanks to the application of color-neutral filters. Three levels of indoor temperature were analyzed in combination with three levels of daylight illuminance, following a mixed-design. As a consequence, each participant experienced one temperature level and the three daylight conditions (non-glary as they were sitting close to the north façade), presented in a randomized order across participants, for a total of three hours. Considering the relatively long exposure, experiments were performed only under clear sky conditions to avoid uncontrolled illuminance changes during each experimental session.

\subsection{Participants}

A total of 122 participants took part in the experiment. The responses of 38 of them were not included in the analysis due to unexpected illuminance variations during experimental sessions. As a result, only the responses of 84 participants were analyzed. Participants were equally distributed across temperature levels (28 participants experiencing each temperature condition), with gender completely balanced (14 women and 14 men in each condition). Participants were recruited from the university community in Lausanne (Switzerland), after a pre-selection process aiming at including people between 18 and 35 years old, with a BMI between 18 and $25 \mathrm{~kg} / \mathrm{m}^{2}$, that did not make use of drugs or abused of alcohol, in good health and which were French speaking (mother tongue or at least $\mathrm{C} 2$ level). Participants that might have been aware of the research aim (due to contacts with the laboratory through lectures, visits and prior experiments), as well as students of disciplines related to the indoor environment (i.e., architecture and civil and environmental engineering) were excluded from the study to avoid biased responses. Written informed consent was obtained previous to the experiment.

\subsection{Stimuli}

The investigation tested the effect of two types of indoor environmental stimuli on participants' performance, namely daylight illuminance and indoor temperature. The two stimuli were presented in a randomized order following a $3 \times 3$ full factorial mixed-design, testing three levels of daylight illuminance (low, medium and high) and three levels of temperature $(19,23$ and $27^{\circ} \mathrm{C}$ ). The daylight illuminance was the within-subject stimulus, experienced by all participants in a randomized order, while the indoor temperature was the between-subject stimulus as each participant experienced only one of the three possible thermal conditions. The three levels of daylight illuminance were achieved with the use of color-neutral filters applied on the glazing of the north and the south openings of the test room, leading to a visible transmittance of $7 \%$ (low illuminance), $30 \%$ (medium illuminance) and $75 \%$ (high illuminance) depending on the glazingfilter combination. The three transmittance combinations resulted in an average of $140 \pm 20$ lux (low), $610 \pm 90$ lux (medium) and 1440 \pm 180 lux (high), measured at the desk level of each participant (for a detailed description of the experimental room see Chinazzo et al. - 2018). The thermal conditions, set and controlled with a radiant system installed in all opaque surfaces of the test room, were chosen to achieve a comfortable condition and two slightly uncomfortable ones (slightly cold and slightly warm) in relation to the clothing ensemble requested to wear by participants (i.e., 0,7 clo - EN ISO, 2006). 


\subsection{Dependent measures}

Participants' performance was evaluated using objective and subjective methods. To evaluate the objective cognitive performance, three types of paper-based tasks were repeated at different stages of the experiment. One task evaluated the distributed visual attention (Tsai Partington), another the sustained vigilance (d2 test) and the last the logical reasoning (Baddeley test). Then, through a questionnaire, participants expressed their subjective evaluations about the tasks performed, as well as their personal state at the moment of the test (e.g., alertness). These two types of evaluations (i.e., objective and subjective) are described in the following subsections. A description of the indoor environmental stimuli measured throughout each experimental session is also reported in the last subsection.

\subsubsection{Objective performance measurements}

The ten-minute test battery was composed by three paper-based performance tests investigating different types of office tasks, chosen from those reported in other investigations on the influence of indoor environmental factors on cognitive performance. These tests were chosen instead of others (e.g., Stroop test) because they were fast and easy to be implemented and corrected on paper as, in this experiment, other sources of light such as computers were avoided. Text typing test, despite having been reported to be a good performance indicator as it is a representative of realistic office tasks and, for this reason, it is free from learning effects (due to the fact that most office workers perform it every day) (Wargocki, 1998), was not used as it involved the use of computers. The three tasks were presented in the same order in all the five experimental conditions (i.e., beginning, three daylight illuminance levels and end - see "2.4. Procedure" section). Different versions of the same test were presented in a randomized order across participants to reduce the learning effect. For each task, a score indicator and an error indicator were calculated.

The Tsai-Partington test (Ammons, 1955), evaluating the distributed visual attention, consists of 30 numbers (from 1 to 99 ) randomly scattered over the page. The participants' task was to trace a line as rapidly as possible through the numbers in ascending order starting from the "Start" sign in the center of the page, within 40 seconds. The Tsai-Partington score used in the analysis consisted of the total number of links minus the incorrect or missing links. The sum of these two last results consisted in the Tsai-Partington error.

The $\mathrm{d} 2$ test developed by Brickenkamp (Brickenkamp, 2002) was used to evaluate the sustained vigilance and concentration. It consists of 14 lines, each containing 47 symbols, either a letter " $d$ " or " $p$ " with either one or two marks (either "or ") above and/or below each letter. The participants' task was to tick as rapidly as possible only the letter $d$ with two marks, either below or above, within 15 seconds per line. Several indicators could be derived from this test. In this study, we calculated the $\mathrm{d} 2$ test score based on equation 1 :

$$
d 2 \text { test score }=(G Z-2 \cdot F 1)+C P
$$

where

$G Z$ is the number of marked letters (either correct or non-correct);

$F 1 \quad$ is the omission error (number of missed d with 2 marks);

$C P \quad$ is the number of correct marks (d with 2 marks) minus the confusion error (number of marks on the wrong letter);

The considered equation takes into consideration both the global performance indicator (GZ$2^{*} \mathrm{~F} 1$ ) and the concentration performance indicator (CP). The $\mathrm{d} 2$ test error indicator, on the other hand, was calculated by summing up the omission and the confusion errors.

The Baddeley test (Baddeley, 1968), measuring the logical reasoning, consists of a sequence of short sentences followed by a pair of letters ("AB" or "BA"). The sentences describe correctly or incorrectly the order of the letters following each statement (e.g., " $A$ is followed by $B$ - AB" is correct or "B does not precede A - BA" is incorrect). The participants' task consisted in indicating whether each short sentence correctly described the order of the letter pair or not, by ticking the box "true" or "false" next to each sentence and pair of letters. They had to evaluate as many sentences as possible starting from the first sentence and without skipping any, within 3 minutes. The Baddeley test score was calculated as the total number of sentences evaluated 
minus the errors and missing evaluations. The sum of these two last results consisted in the Baddeley test error.

Each of the three scores was normalized according to the maximum possible result of the test and multiplied by 100 , to allow comparability across measurements. The obtained values (indicated as test performance in \%) are the ones used in the statistical analysis and graphs.

\subsubsection{Subjective responses}

A series of questions were asked to participants at the end of each performance test battery to record their subjective performance evaluations as well as their personal state. The questionnaire was distributed on a tablet. The questions, presented in the same order, are summarized in Table 1. For each of the questions, an evaluation name is given to facilitate the result and discussion sections. The responses to each question were evaluated according to the numerical scale indicated in bracket in Table 1.

Table 1 - Subjective performance evaluation and personal state questions

\begin{tabular}{|c|c|c|}
\hline $\begin{array}{l}\text { Evaluation } \\
\text { name }\end{array}$ & Question & Response scale \\
\hline $\begin{array}{l}\text { Alertness } \\
\text { (Karolinska } \\
\text { Sleepiness } \\
\text { Scale - KSS) }\end{array}$ & $\begin{array}{l}\text { Please indicate how you feel at this } \\
\text { moment: }\end{array}$ & $\begin{array}{l}\text { Extremely alert (1), } \\
\text { Very alert (2), Alert (3), Rather } \\
\text { alert (4), Neither alert nor sleepy } \\
\text { (5), Some signs of sleepiness } \\
(6), \text { Sleepy, but no effort to keep } \\
\text { awake ( } 7) \text {, Sleepy, some effort } \\
\text { to keep awake (8), Very sleepy, } \\
\text { great effort to keep awake, } \\
\text { fighting sleep (9) }\end{array}$ \\
\hline Energy & \multirow{3}{*}{$\begin{array}{l}\text { Please indicate how you feel at this } \\
\text { moment. For each pair, put a check } \\
\text { mark closer to the adjective which you } \\
\text { believe to describe your feelings better: }\end{array}$} & Stimulated (1) - Relaxed (7) \\
\hline Excitement & & Excited (1) - Calm (7) \\
\hline Fatigue & & Wide awake (1) - Sleepy (7) \\
\hline $\begin{array}{l}\text { Perceived } \\
\text { concentration }\end{array}$ & $\begin{array}{l}\text { How able are you to concentrate right } \\
\text { now (on a scale of } 1 \text { to } 10, \text { where } 1 \text { is } \\
\text { not able to concentrate at all and } 10 \text { is } \\
\text { fully able to concentrate)? }\end{array}$ & $(1)-(10)$ \\
\hline $\begin{array}{l}\text { Work } \\
\text { evaluation }\end{array}$ & $\begin{array}{l}\text { With reference to the tasks you just } \\
\text { completed, the work was: }\end{array}$ & $\begin{array}{l}\text { Very easy }(0) \text { - Very difficult } \\
(100)\end{array}$ \\
\hline $\begin{array}{l}\text { Effort } \\
\text { evaluation }\end{array}$ & $\begin{array}{l}\text { With reference to the tasks you just } \\
\text { completed, your effort was: }\end{array}$ & Very low (0) - Very high (100) \\
\hline $\begin{array}{l}\text { Time } \\
\text { pressure }\end{array}$ & $\begin{array}{l}\text { With reference to the tasks you just } \\
\text { completed, the pressure caused by the } \\
\text { time allowed to complete the tasks was: }\end{array}$ & Very low (0) - Very high (100) \\
\hline $\begin{array}{l}\text { Performance } \\
\text { evaluation }\end{array}$ & $\begin{array}{l}\text { With reference to the tasks you just } \\
\text { completed, your performance was: }\end{array}$ & Mediocre (0) - Excellent (100) \\
\hline
\end{tabular}

\subsubsection{Indoor environmental factors' measurements}

Indoor environmental conditions were continuously measured over the full duration of the experimental sessions. Indoor temperature was measured through air and globe temperature measurements at 4 different heights for each participant, in agreement with the EN ISO 7726 standard (EN ISO, 2002). Horizontal illuminance was recorded at the desk level with two luxmeters located on the left and the right of each participant (roughly at $40 \mathrm{~cm}$ per side). Indoor temperature and illuminance measurements were relied upon to verify that the targeted indoor 
environmental conditions were maintained and that they were equally distributed across experimental conditions (i.e., illuminance levels in the three daylight conditions were constant at the three temperature levels, and indoor temperature in each thermal condition was constant for the three daylight illuminance levels).

Other indoor environmental factors were measured in the middle of the room at a 1-min sampling rate: relative humidity, $\mathrm{CO}_{2}$ concentration and air velocity. The measured values were used to verify constant conditions in all daylight illuminance conditions, as well as acceptable levels throughout the experimental sessions. The raw data referring to the measurements were not included in the statistical analysis as only categorical factors were considered. Moreover, relative humidity and air velocity had very small variations across conditions. Only $\mathrm{CO}_{2}$ concentration varied substantially during each experimental session, but its variation was totally confounded with the order of presentation of the experimental conditions as it increased over time (i.e., $\mathrm{CO}_{2}$ concentration was higher in the second daylight condition compared to the first one, as well as in the third daylight condition compared to the second one). Figure 1 shows this increase of $\mathrm{CO}_{2}$ concentration over time due to the occupation of the room by participants and the researcher. Due to this systematic increase over time, $\mathrm{CO}_{2}$ concentration was not included in the analysis. It must be noted that the $\mathrm{CO}_{2}$ concentration was slightly higher compared to the recommended threshold (1030 ppm - American Society of Heating, Refrigerating and AirConditioning Engineers, 2013), but it occurred in all daylight illuminance conditions. Moreover, the recorded values are representative of concentrations typically found in office buildings (Seppänen et al., 1999).

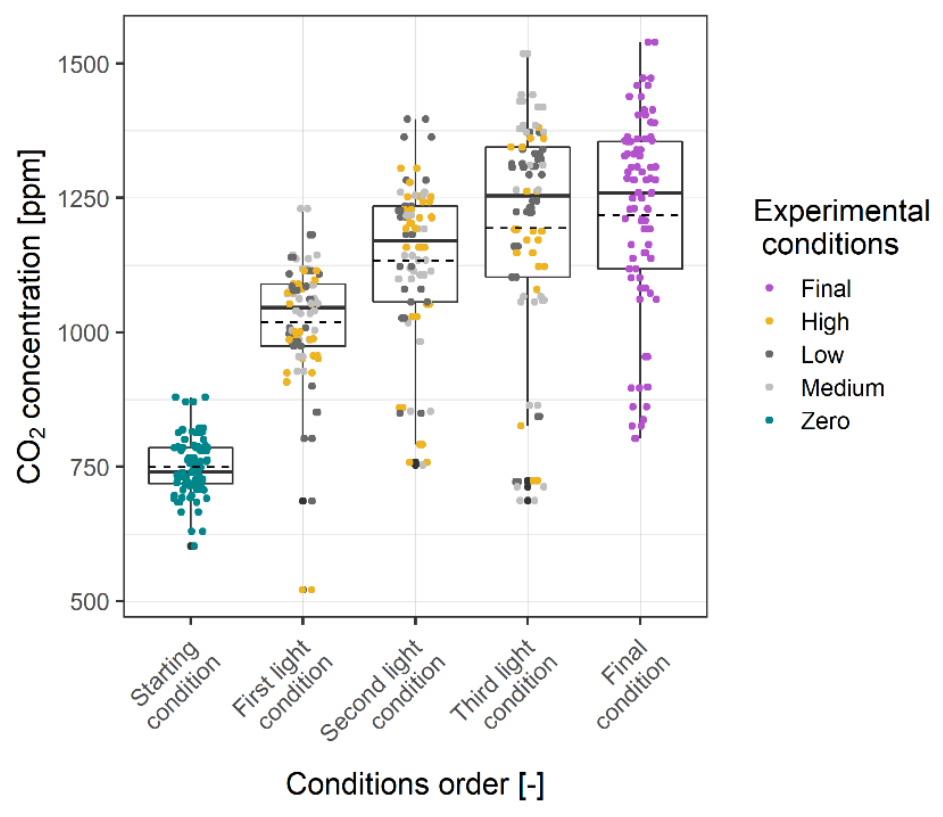

Figure 1 - Boxplot of the indoor air quality measured in each experimental condition, showing an increasing $\mathrm{CO}_{2}$ concentration due to the occupation of the room. The dashed line indicates the mean value, and the thick black one the median value. Points illustrate the measured values according to the experimental condition

\subsection{Procedure}

Two participants at a time took part in the experiment, which lasted approximately 3 hours. The first 45 minutes were used for thermal and metabolic adaptation to one of the three experimental temperatures. In this phase, referred to as "starting condition", participants were instructed about the goal of the experiment. They were told that their performance will be evaluated in relation to the indoor environment, without specifying if the environment changes nor in which way. In this phase, each performance task was explained in detail, and a first trial was performed by participants for each task after its explanation, without any time constrain (for this reason, these trials were not used in the evaluation of the performance). Then, participants were told that the same test performance battery, composed by the three tasks, will be repeated 
five times over the course of the experiment, followed by a questionnaire about their selfestimated performance in relation to the tasks completed.

The first performance test battery was performed before the exposure to the daylight levels, in the starting condition, in which electric light was turned on (at 400 lux at the desk level, 3800 $\mathrm{K}$ ) and daylight was blocked from entering the test room with blackout curtains. This session was used a training phase (this time with the time constrain), to limit the learning effect detected in past investigations (Ammons, 1955; Sleegers et al., 2013; Valančius and Jurelionis, 2013; Wargocki, 1998). Then, the same tasks were performed during each of the three daylight exposures, experienced in a randomized order across participants. The fifth round was conducted at the end of the daylight exposure, in the "final condition", under the same electric light conditions as at the beginning. Each daylight condition lasted approximately 30 minutes and before the start of the performance test, participants had the time to adapt to the visual condition by visually exploring the room for few minutes $(\sim 2 \mathrm{~min})$ and by reading a text for 5 minutes. To make sure participants were actively engaged in the reading task, they were told that some questions regarding the text would have been part of the performance evaluation. However, data related to such responses will not be analyzed as responses were closely related to the version of the text distributed. A break time of 10 minutes before each daylight condition, in which participants were told to relax while blindfolded and while listening to music, allowed the change of the filters on the south and north openings.

In each temperature condition, half of the participants were told about the presence of a prize (a gift card), promised to the person with the total highest score (across the five test batteries and the three tasks in each of them). The other half was not informed about the prize.

As daylight was the only source of light for the greatest part of the experiment (except the starting and final conditions), experiments were conducted in both mornings and afternoons, starting between $8: 30$ and 9:00, or between $12: 30$ and 13:00, respectively, to maximize the experimental time in sunny conditions. Experiments took place from October 2017 to December 2017 and from April 2018 to June 2018, in a discontinuous way as only days with clear sky conditions were selected.

The study was approved by the Cantonal Ethics Committee of the Canton Vaud, Switzerland (CER-VD, ref No. 2016-01115), and adhered to the principles of the Declaration of Helsinki.

\subsection{Statistical analysis}

Objective and subjective performance indicators were analyzed with a linear mixed effects model to study the main effect of daylight illuminance and indoor temperature, as well as that of their interaction. This type of analysis takes into consideration repeated measurements of the performance tasks and of the subjective measures by modeling different intercepts for each participant (random effect factors of the model). In case of significant interactions, additional analyses were conducted at each temperature level, otherwise the interaction term was removed from the model. In case of significant main effect of daylight illuminance or indoor temperature, post-hoc tests were conducted to evaluate all possible pairwise comparisons across the three levels of each factor, applying the multiplicity adjustment Tukey's HSD test to prevent from Type I error (Coolican, 2014). For all responses, additional covariates were included in the model to consider factors that were balanced in the analysis and/or that could have influenced the results: experimental time (morning or afternoon sessions), gender, prize and order of presentation of daylight condition.

All the analyses were performed with the dataset referring to the daylight conditions, hence excluding the starting and the final conditions. The statistical tests were performed using the software R (R Core Team, 2017) with the RStudio integrated development environment. The significant level for all analyses was set to 0,05 .

\section{Results}

Results are reported in two subsections. In the first one, the effects of visual and thermal conditions on objective performance tests are analyzed, together with those of the considered covariates included in the analysis. In the second, the effects on subjective responses are discussed. 


\subsection{Effects on objective performance tests}

Table 2 summarizes the significant effects of the modeled factors for each of the three performance tasks, that are considered both in terms of score and error. Only the significant covariates are reported in the column "Other factors". As can be seen, the interaction term between daylight illuminance levels and indoor temperatures was never a significant factor, for all of the objective performance tests. Similarly, indoor temperature did not affect the objective task scores, nor the errors. Daylight illuminance resulted as a significant factor only for the $\mathrm{d} 2$ test score $(F(2,243)=3,25, p=0,041)$. However, considering the relatively high $p$ value and the fact that post-hoc tests indicated a (very small) significant difference only between the medium and the high daylight illuminance conditions (estimated difference $=0,74, \mathrm{t}(243)=-$ $2,45, p=0,040)$, the result can reasonably be considered an effect of chance. As a result, we can consider that objective performance results did not differ according to the daylight illuminance levels, with similar outcomes under the low, medium and high daylight illuminance conditions. The same result occurred at all the three temperature levels investigated.

The only significant factor influencing the objective performance indicators, as indicated in Table 2, was the order of presentation of the daylight conditions (all p's $<0,001$ ). This result occurred for all the normalized scores of the three tests and for the $d 2$ test error $(F(2,243)=$ $4,26, p=0,015)$. As post-hoc tests indicated an increasing score across all performance test trials (for all tasks) and a decreasing number of errors for the d2 test, results imply a learning effect. Figure 2 illustrates the learning effect for the three normalized performance task scores, for the five performance test batteries (hence including the starting and final conditions, not analyzed in the statistical analysis).

Table 2 - Statistical results - objective performance tests. Significant effect indicated with "**” for $p<0,05$, “***" for $p<0,01$, “****" for $p<0,001$. The sign ". " indicates no significant effect

\begin{tabular}{|l|l|l|l|l|l|}
\hline \multicolumn{2}{|c|}{} & Illuminance & Temperature & III $\times$ Temp & Other factors \\
\hline $\begin{array}{l}\text { Tsai } \\
\text { Partington } \\
\text { test }\end{array}$ & Score & - & - & - & $\begin{array}{l}\text { Order of presentation } \\
(\uparrow \text { over trials })\end{array}$ \\
\cline { 2 - 6 } & Error & - & - & - & - \\
\hline $\begin{array}{l}\text { Baddeley } \\
\text { test }\end{array}$ & Score & - & - & - & $\begin{array}{l}\text { Order of presentation } \\
(\uparrow \text { over trials })\end{array}$ \\
\cline { 2 - 7 } & Error & - & - & - & - \\
\hline \multirow{2}{*}{ d2 test } & Score & $\begin{array}{l}* \text { (result of } \\
\text { chance) }\end{array}$ & - & - & $\begin{array}{l}\text { Order of presentation } \\
(\uparrow \text { over trials })\end{array}$ \\
\cline { 2 - 6 } & Error & - & - & - & $\begin{array}{l}\text { Order of presentation } \\
(\downarrow \text { over trials })\end{array}$ \\
\hline
\end{tabular}




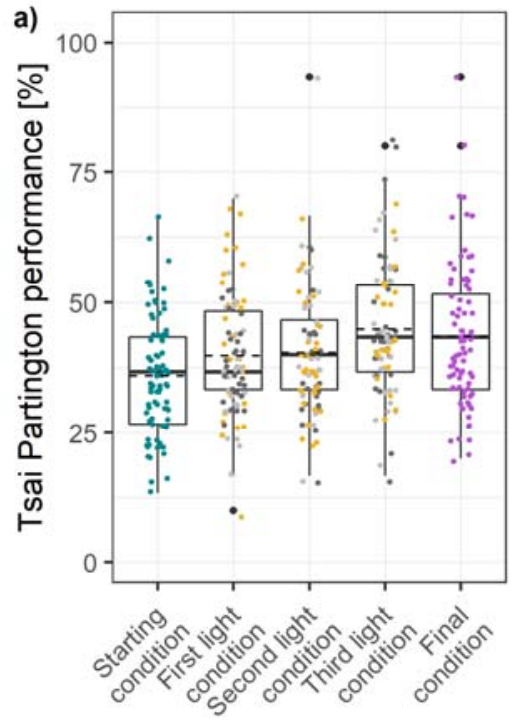

Conditions order [-]

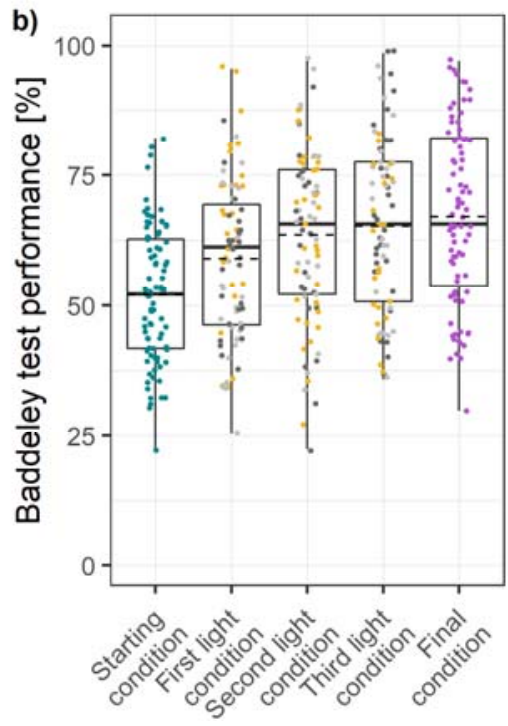

Conditions order [-]

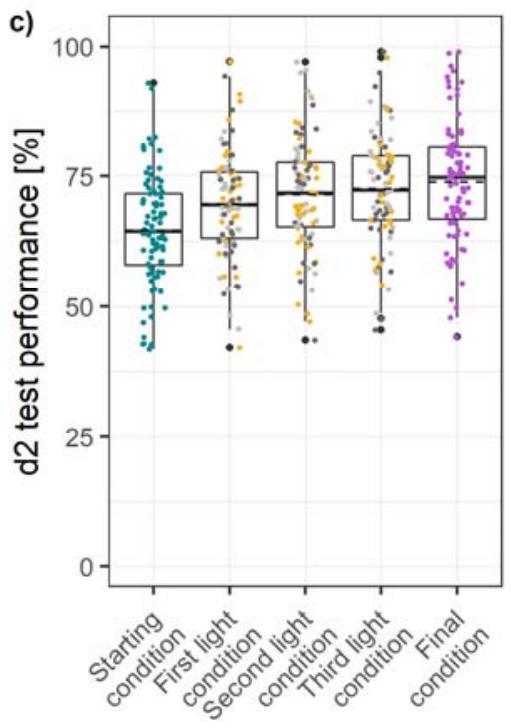

Conditions order [-]

Experimental conditions - Final · High · Low . Medium * Zero

Figure 2 - Boxplot of the performance test results for the three tasks (a- Tsai Partington; bBaddeley test; $c-d 2$ test) in each experimental condition, showing a learning effect for all the tasks. The dashed line indicates the mean value, and the thick black one the median value. Points illustrate the measured values according to the experimental condition

\subsection{Effects on subjective responses}

Table 3 summarizes the results of the statistical analysis performed on the subjective responses of participants, reported after the completion of the objective performance test in each daylight condition. Also in this case, the interaction term and the temperature were never significant factors for the determination of the subjective responses. Contrary to the results for the objective performance tasks, the order of presentation did not affect the subjective responses.

A significant difference under exposure to different daylight illuminance levels was indicated only for perceived concentration $(F(2,243)=3,59, p=0,029)$. Post-hoc tests indicated that the self-reported concentration was significantly lower under the low illuminance exposure compared to the medium one (estimated difference $=0,36, \mathrm{t}(243)=-2,39, \mathrm{p}=0,046$ ) and almost significantly lower under the low illuminance level compared to the high one (estimated difference $=0,34, t(243)=-2,23, p=0,068)$, independently of the thermal condition. The perceived concentration was significantly affected by the time of day factor $(F(1,243)=5,14$, $p=0,025)$, with participants perceiving their concentration lower in the afternoon compared to the morning (estimated difference $=0,55, \mathrm{t}(243)=-2,26, \mathrm{p}=0,025)$. Time of day was also a significant factor for perceived fatigue $(F(1,243)=6,99, p=0,009)$, with people more tired in the afternoon compared to the morning (estimated difference $=0,55, t(243)=-2,64, p=0,009$ ), and for effort evaluation $(F(1,243)=5,1, p=0,026)$, with a perceived higher effort in the morning compared to the afternoon (estimated difference $=7,1, t(243)=-2,25, p=0,026$ ). The effort evaluation was influenced by the prize factor as well $(F(1,243)=9,81, p=0,002)$, with a perceived higher level of effort whenever the prize was introduced to participants (estimated difference $=9,3, t(243)=-3,13, p=0,002)$. The prize was also a significant factor for the work evaluation $(F(1,243)=4,84, p=0,030)$, with an easier work estimated in the absence of the prize (estimated difference $=5,88, \mathrm{t}(243)=-2.2, \mathrm{p}=0,030$ ). Finally, the self-estimated performance evaluation was affected by the gender of participants $(F(1,243)=6,38, p=0,013)$ as women underestimated their performance in comparison to men (estimated difference $=6,6$, $t(243)=-2,52, p=0,013)$. 
Table 3 - Statistical results - subjective responses. Significant effect indicated with "**" for $p<$ 0,05 , “**”" for $p<0,01$, "“****” for $p<0,001$. The sign "-" indicates no significant effect

\begin{tabular}{|l|l|l|l|l|}
\hline & Illuminance & Temp & III x Temp & Other factors \\
\hline Alertness & - & - & - & \\
\hline Energy & - & - & - & \\
\hline Excitement & - & - & - & \\
\hline Fatigue & - & - & - & Morning/ Afternoon ** $(\uparrow$ in afternoon $)$ \\
\hline $\begin{array}{l}\text { Perceived } \\
\text { concentration }\end{array}$ & $\begin{array}{l}{ }^{*}(\downarrow \text { in low } \\
\text { illuminance })\end{array}$ & - & - & Morning/ Afternoon * $(\downarrow$ in afternoon $)$ \\
\hline Work evaluation & - & - & - & Prize $^{*}(\downarrow$ without prize $)$ \\
\hline Effort evaluation & - & - & - & $\begin{array}{l}\text { Morning/ Afternoon }{ }^{*}(\uparrow \text { in morning }) \\
\text { Prize }^{* *}(\uparrow \text { with prize })\end{array}$ \\
\hline Time pressure & - & - & - & - \\
\hline $\begin{array}{l}\text { Performance } \\
\text { evaluation }\end{array}$ & - & - & - & Gender * $(\downarrow$ for women $)$ \\
\hline
\end{tabular}

\section{Discussion}

Very different outcomes were found for the objective and the subjective performance indicators.

The results of the objective performance tasks varied only in relation to the order of trial presentation, showing a learning effect as reported in some past investigations (Ammons, 1955; Sleegers et al., 2013; Valančius and Jurelionis, 2013; Wargocki, 1998). The presence of the learning effect might be due to the too few "training" tests performed prior to the "real" tests in the daylighting conditions (in this experiment participants performed one training test without time constrain and one with time constrain), to the short exposure time and to the types of test used. These latter, in fact, despite investigating different types of cognitive performance that can be associated with office tasks, might not be representative of the actual tasks performed in offices. For future investigations, other types of test are suggested. In particular, whenever possible to use a computer, the text typing is recommended as it is a typical office task free of learning effect and has been shown to vary according to indoor conditions (Wargocki, 1998). Without the presence of learning effects (and eventually longer exposure time), indoor conditions might result as significant factors affecting objective task performance. In particular, it is hypothesized that low illuminance levels might reduce the performance of people.

This hypothesis is corroborated by the outcome related to participants' perceived concentration, which varied in response to daylight illuminance levels, as a lower self-estimated concentration was reported in the low illuminance condition. This occurred independently of the thermal conditions, indicating no interactions between daylight illuminance and temperature. A low selfestimated concentration due to low illuminance conditions, therefore, might affect building occupants' objective performance.

Besides the perceived concentration, the other subjective responses were not affected by daylight illuminance, indoor temperature, or their interaction. On the other hand, other factors influenced some of the responses. Time of day affected fatigue, perceived concentration and effort evaluation. The presence of a prize influenced the work and the effort evaluations. Finally, the gender affected the performance evaluation, with women underestimating their performance in comparison to men.

\section{Conclusion}

This study reported the combined effects of daylight illuminance and indoor temperature levels on objective and subjective cognitive performance. Experimental investigations were conducted in an office-like test room to be able to change and control the indoor conditions, and to study the effects of daylight without introducing confounding variables as in field studies. 
Results did not indicate significant differences between the low, medium and high daylight illuminance levels for all the dependent variables investigated, except for the self-evaluated level of concentration, which resulted lower under the low daylight illuminance exposure. For all the other objective and subjective performance responses we did not find a significant decrease in performance under low daylight illuminance or an increase of it under high daylight illuminance. Moreover, results were not affected by indoor temperature nor by its interaction with daylight illuminance level.

It must be remarked that, especially for the objective tasks, responses might have been affected by the too few trial tests performed prior to the real tests, the short exposure time and the type of test used. Considering the effect of daylight illuminance levels on perceived concentration, it was hypothesized that responses of a different type of task (e.g., text typing, free of learning effect) or after a longer exposure time (with repeated test trials), might be affected by daylight illuminance levels as well, with a decreased performance in a poorly lit environment. Moreover, such illuminance influence would occur independently of the thermal conditions inside the room. Further investigations are necessary to confirm these hypotheses.

\section{References}

Al Horr, Y., Arif, M., Kaushik, A., Mazroei, A., Katafygiotou, M., Elsarrag, E., 2016. Occupant productivity and office indoor environment quality: A review of the literature. Build. Environ. 105, 369-389. https://doi.org/10.1016/j.buildenv.2016.06.001

American Society of Heating, Refrigerating and Air-Conditioning Engineers, 2013. ANSI/ASHRAE Standard 62.1-2013 - Ventilation for Acceptable Indoor Air Quality. The Standards For Ventilation And Indoor Air Quality.

Ammons, C.H., 1955. Task for the Study of Perceptual Learning and Performance Variables. Percept. Mot. Skills 5, 11-14. https://doi.org/10.2466/pms.1955.5.g.1

Baddeley, A.D., 1968. A 3 min reasoning test based on grammatical transformation. Psychon. Sci. 10, 341-342.

Balazova, I., Clausen, G., Wyon, D.P., 2007. The influence of exposure to multiple indoor environmental parameters on human perception, performance and motivation, in: Proceedings of CLIMA.

Baron, R.A., Rea, M.S., Daniels, S.G., 1992. Effects of indoor lighting (illuminance and spectral distribution) on the performance of cognitive tasks and interpersonal behaviors: The potential mediating role of positive affect. Motiv. Emot. 16, 1-33. https://doi.org/10.1007/BF00996485

Brickenkamp, R., 2002. Aufmerksamkeits-Belastungstest d2. Gött. Hogrefe.

Chinazzo, G., 2019. Daylight and temperature in buildings: interaction effects on human responses. Ecole polytechnique fédérale de Lausanne, Lausanne, Switzerland.

Chinazzo, G., Wienold, J., Andersen, M., 2019a. Daylight affects human thermal perception. Unpubl. Results.

Chinazzo, G., Wienold, J., Andersen, M., 2019b. Influence of indoor temperature and daylight illuminance levels on visual perception. Unpubl. Results.

Chinazzo, G., Wienold, J., Andersen, M., 2018. Combined effects of daylight transmitted through coloured glazing and indoor temperature on thermal responses and overall comfort. Build. Environ. https://doi.org/10.1016/j.buildenv.2018.08.045

Clements-Croome, D., 2006. Creating the Productive Workplace. Taylor \& Francis.

Coolican, H., 2014. Research Methods and Statistics in Psychology, 6th ed. Psychology Press.

EN ISO, 2006. 7730-2006: Ergonomics of the thermal environment-Analytical determination and interpretation of thermal comfort using calculation of the PMV and PPD indices and local thermal comfort criteria. BSI, London, UK. 
EN ISO, 2002. 7726-2001: Ergonomics of the thermal environment - Instruments for measuring physical quantities. BSI, London, UK.

Furnham, A., Strbac, L., 2002. Music is as distracting as noise: the differential distraction of background music and noise on the cognitive test performance of introverts and extraverts. Ergonomics 45, 203-217.

Heschong Mahone Group, 2003. Windows and Classrooms: A Study of Student Performance and the Indoor Environment. California Energy Commission.

Hygge, S., Knez, I., 2001. Effects of Noise, Heat and Indoor Lighting on Cognitive Performance and Self-Reported Affect. J. Environ. Psychol. 21, 291-299. https://doi.org/10.1006/jevp.2001.0222

Knez, I., Hygge, S., 2002. Irrelevant speech and indoor lighting: effects on cognitive performance and self-reported affect. Appl. Cogn. Psychol. Off. J. Soc. Appl. Res. Mem. Cogn. 16, 709-718.

Lan, L., Lian, Z., 2009. Use of neurobehavioral tests to evaluate the effects of indoor environment quality on productivity. Build. Environ. 44, 2208-2217. https://doi.org/10.1016/j.buildenv.2009.02.001

Liebl, A., Haller, J., Jödicke, B., Baumgartner, H., Schlittmeier, S., Hellbrück, J., 2012. Combined effects of acoustic and visual distraction on cognitive performance and wellbeing. Appl. Ergon., Special Section on Product Comfort 43, 424-434. https://doi.org/10.1016/j.apergo.2011.06.017

Lin, C.-C., 2014. Effect of noise intensity and illumination intensity on visual performance. Percept. Mot. Skills 119, 441-454.

Löfberg, H.A., Löfstedt, B., Nilsson, I., Wyon, D.P., 1975. Combined temperature and lighting effects on the performance of repetitive tasks with differing visual content, in: Proceedings of the 18th CIE Conference.

R Core Team, 2017. R: A Language and Environment for Statistical Computing. R Foundation for Statistical Computing, Vienna, Austria.

Reinten, J., Braat-Eggen, P.E., Hornikx, M., Kort, H.S.M., Kohlrausch, A., 2017. The indoor sound environment and human task performance: A literature review on the role of room acoustics. Build. Environ. 123, 315-332. https://doi.org/10.1016/j.buildenv.2017.07.005

Seppänen, O.A., Fisk, W.J., Mendell, M.J., 1999. Association of ventilation rates and CO2 concentrations with health andother responses in commercial and institutional buildings. Indoor Air 9, 226-252.

Sleegers, P., Moolenaar, N., Galetzka, M., Pruyn, A., Sarroukh, B., van der Zande, B., 2013. Lighting affects students' concentration positively: Findings from three Dutch studies. Light. Res. Technol. 45, 159-175. https://doi.org/10.1177/1477153512446099

Torresin, S., Pernigotto, G., Cappelletti, F., Gasparella, A., 2018. Combined effects of environmental factors on human perception and objective performance: A review of experimental laboratory works. Indoor Air.

Valančius, R., Jurelionis, A., 2013. Influence of indoor air temperature variation on office work performance. J. Environ. Eng. Landsc. Manag. 21, 19-25. https://doi.org/10.3846/16486897.2012.721371

Veitch, J.A., 1990. Office noise and illumination effects on reading comprehension. J. Environ. Psychol. 10, 209-217.

Wargocki, P., 1998. Human perception, productivity and symptoms related to indoor air quality. Centre for Indoor Environment and Energy, Department of Energy Engineering. 